\title{
Filtered orthogonal frequency division multiplexing for improved 5G systems
}

\author{
Ammar A. Sahrab, Alaa Doohee Yaseen \\ Department of Electrical Engineering, Al-Mustansiriyah University, Baghdad, Iraq
}

\begin{tabular}{l}
\hline Article Info \\
\hline Article history: \\
Received Dec 5, 2020 \\
Revised Mar 29, 2021 \\
Accepted Jun 17, 2021 \\
\hline
\end{tabular}

\section{Keywords:}

Blackman window

CP-OFDM

f-OFDM

Hamming window

Hanning window

\begin{abstract}
Wireless communications became an integrated part of the human life. Fifth generation $(5 \mathrm{G})$ is the modern communication which provides enhanced mobile broadband (eMBB), ultra reliable low latency communications (URLLC), and massive machine type communication (mMTC). Thus, 5G have to provide coverage to multi-numerology devices, therefore, modulation and access schemes are suggested in the literature such as cyclic prefix orthogonal frequency division multiplexing (CP-OFDM) and filtered OFDM (f-OFDM). CP-OFDM suffers from the high out of band emission which limited the multi-numerology applications. In f-OFDM, the out of band emission can be suppressed to an accepted extent such that different numerologies can be coexisting. On the other hand, f-OFDM can be more improved by using a proper filtering approach. In this paper three different filters are suggested based windowed-sinc function; Hanning, Hamming, and Blackman. Simulation results show that the proposed filters are promising for high spectral efficiency and out of band emission rejection. Furthermore, the bit error rate, error vector magnitude, and power spectral density are further improved with respect to CP-OFDM scheme but some trade-off is present. Overall, the suggested windowed-sinc filters are outperforming the traditional CP-OFDM. As a conclusion, the suggested windnowed-sinc filters have no limitations on the modulation order or the number of subcarriers utilized in the system.
\end{abstract}

This is an open access article under the CC BY-SA license.

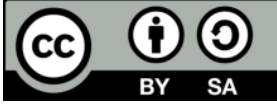

\section{Corresponding Author:}

Alaa Doohee Yaseen

Department of Electrical Engineering

Al-Mustansiriyah University

Baghdad, Palestine Street 10064, Iraq

Email: freeccsud@gmail.com

\section{INTRODUCTION}

Previous wireless generations, particularly the 4th generation (4G), is deployed worldwide. During technology development, numerous services are demanded, including internet of things (IoT) [1]. Massive machine type communication (mMTC) [2] are critical devices that demand fast data rate with sporadic transmission nature [3]. Thus, beside the traditional voice origination call, more services have to be supported; such as video streaming and virtual reality applications [4]. Long term evolution (LTE) and LTE-advanced (LTE-A) which is the technology developed for $4 \mathrm{G}$ wireless systems became uncapable to support these various numerologies. In fact, LTE based systems are built around cyclic prefix-orthogonal frequency division multiplexing (CP-OFDM). The subcarrier spacing in CP-OFDM is fixed, which leads to one-only numerology. The term numerology stands for subcarrier spacing, as $\Delta \mathrm{f}=15 \mathrm{kHz} \times 2 \mu$, where $\mu$ is called numerology [5]. The 4G, as mentioned above, has fixed numerology which is $\mu=0$, in other words 
subcarrier spacing $\Delta \mathrm{f}=15 \mathrm{kHz}$. To support the various services requirements, 5 th generation (5G) has been designed to employ various numerologies, in which different services can be utilized.

In other words, technology continued to be developed, then novel frameworks are invented which may require access to the wireless networks. That is why extra mandated loads have to be available by the wireless network. The traditional voice origination calls and data transferring will be alongside new services, which are revealed every day. On account of this, and beyond 4G, 5G should be capable to deliver different signal forms to cover all new services. Wireless sensor networks are one of the mMTC which circumvents messages of long format, moreover IoT are another type of systems which avoids long messages [6], thus, essential modification to the traditional signal, represented by 4G LTE signals, are mandatory to tackle the coming problems [7]. Customizable waveform-shapes became obligatory to manage the vital specifications. On the other hand, additional information for synchronization is necessary in $4 \mathrm{G}$, which must be released in $5 \mathrm{G}$, this leading to asynchronized communication [8].

The conventional CP-OFDM, which is the modulation and access scheme employed by 4G LTE wireless system, is incompetent to cover the above necessities. In CP-OFDM, the out of band (OOB) emission is significantly effective in the time-domain leading to necessary synchronizations both in time and frequency, because the $\mathrm{OOB}$ emission generated interference with neighbor channels [9]. Strained synchronization emerging additional time (latency) for the system to work properly, this increased latency necessitates more power consumption, therefore, mMTC cannot be deployed with $4 \mathrm{G}$ system because mMTC operates with limited life-time battery [10].

On the other hand, channel estimation is worth to mention here, since it is an important part of the data recovery process at the receing end. Different optimization schemes are listed in the literature. However, two main categories for channel estimation can be said as blind and non-blind techniques. The first category does not send pilot carriers as a reference signal, while the second category does send these pilot symbols as reference signals to aid the receiver to recover the data properly. The non-blind category has further two main sub-categories, block-type and comb-type. There are other combinations in the literature, but the most famous are the block and comb types [11]. The block type is used when the channel looks like do not change the state or it is indoor behaviour. While the comb type is sending the pilot carriers with certain spacings. Optimizing these pilot carrier's location is favorable to enhance the system behaviour [12].

Aforementioned that $5 \mathrm{G}$ system designated to operate such that it provides services to eMBB, mMTC, and URLLC. Hence, various numerologies must be provided in order proper operation. Each one of these services has its own numerology, or signal format, signal size, overhead, for the eMBB. Specifically, $\Delta \mathrm{f}$, cyclic prefix (CP) size/duration, and slot/subframe duration. Over gigabits/second data throughput is the part of eMBB communications, for instance, over $20 \mathrm{~Gb} / \mathrm{s}$. Smart homes/cities are examples of the mMTC. While ultra high definition streaming augmented reality, self-driving machines are sexampls of URLLC [8].

As a result, the physical layer of the new system has to be very elastic. Fortunately, signals can be formed flexibly. Thus, the literature is rich of waveforms that fits to the $5 \mathrm{G}$ system but with trade-offs. Namely, filter bank multicarrier (FBMC) [13], universal filtered multicarrier (UFMC), aka universal filtered OFDM (UF-OFDM) [10], generalized frequency division multiplexing [14], and the filtered OFDM (f-OFDM) [15]. These mentioned waveforms are all based on filtering operation. Industry and academia communities paid much attention for such types of waveforms, because filtering-based waveforms are outperforming the conventional CP-OFDM waveform [16]. Each of these filtering-based waveforms have its cons and pros. In particular, subcarrier filtering achieved in FBMC and GFDM have to employ the offset/staggered digital quadrature amplitude modulation (QAM), consequently, these two waveforms are not friendly with multiple input multiple output (MIMO) multiplexing schemes [17], [18]. UFMC waveform outperforms FBMC and GFDM but at the cost of filter size but not comparable to f-OFDM where the filter size is more realistic [15], [19], [20], accordingly, f-OFDM can be the promising based waveform for the 5G wireless systems.

In account of the previous discussion, filter design must be smartly designed in order to not increase latency of complexity to the system. Furthermore, waveforms based on filtering must fit the requirement which are stated previously for the 5G system. Worthy say that CP-OFDM exploits the sinc-function based filter in the frequency domain. The sinc-based filter grieves high sidelobes, which are considerably degrades the system performance. A systematic direct implementable filter can be used to originate the f-OFDM waveform [21]. This filter is called truncated-sinc filter. This filter can be generated by windowing the sincfunction with properly and smartly selected window. Thus, it was shown that windowed-sinc filter based fOFDM outperforms CP-OFDM waveform.

Abdoli used Hanning-window [19], [22]. In another suggestion, Bazzi proposed to use Von Hann window, following the same procedure of Abdoli, however, an outperforming behavior with respect to FBMC and UFMC, but the OOB emission can be recognized significantly [23]. It is necessary and essential to avoid additional computational complexity, since the latency increased, C. An et. al. proposed a technique 
that destroys the latency due to the additional computational complexity, where windowing/filtering is configured according to the location of the filter or the window, thus, four configurated schemes produced but at the cost of the degraded latency [24].

The Gaussian window-based filter proposed in other work aiming to reduce the OOB emission [25]. However, Gaussian based filter destroys the orthogonality between subcarriers, this loss in orthogonality produces intercarrier interference (ICI) as well as intersymbol interference (ISI). On the other hand, the finite impulse response (FIR) is another suggestion to overcome the OOB problem. It is found that the OOB problem was resolved but the computational complexity was degraded significantly [26].

In 2019, a confirmation result of [15], [19] was obtained where the OOB emission still recognized leading to loss of the synchronization [27]. The computational complexity of filtered based waveforms was suggested, which is the singular value decomposition (SVD) methodology [28]. Although the reduced complexity and OOB emission enhancements, the BER of the system was degraded.

In this paper, the physical layer of $5 \mathrm{G}$ wireless systems will be simulated using three different windowed-sinc filters, Hanning, Hamming, and Blackman based filters. The results will be compared fairly with the conventional CP-OFDM in terms of OOB emission rejection, BER improvement performance, error vector magnitude. Specifically speaking, the contribution of this paper is that these filters will be link level simulation and the throughput for 100ms transmission interval will be used with MIMO of eight transmitting and two receiving antennas will be employed for the downlink direction. Such link level implementation has not been achieved in the literature, at least, with the author's point of view. Thus, this paper can be a useful resource for the researchers and academia.

\section{SYSTEM MODEL AND FILTER DESIGN}

The system which will be simulated in this work can be seen in Figure 1. The difference between $\mathrm{f}-\mathrm{OFDM}$ and CP-OFDM is that the filter $f_{i}(n)$ is not applicable in CP-OFDM, that is, both CP-OFDM and f-OFDM can be represented in Figure 1, which is the basic components to generate the physical layer for downlink link-level simulation. The binary stream of each sub-band will be first mapped to one of the QAM orders, according to the payload. Serial to parallel conversion (demultiplexing) is the next step followed by frequency-domain to time-domain conversion using the N-points inverse fast fourier transform (IFFT), then converting back to the serial form using during the parallel to serial block (multiplexing). To combat the multipath channel, the $\mathrm{CP}$ is inserted. The last operation is the filtering operation which will be applied only to generate the f-OFDM waveform as shown in the upper part of Figure 1. The lower part of Figure 1 stands for the receiver end, which follows the reverse operations of the transmitter. The transmitting operation can be expressed mathematically by adjusting various sub-band configurations, which are obtained using distinct numerologies, $i=1,2 \ldots I$, where $I$ represents the number of individual sub-bands,

$$
x(n)=\left[\frac{1}{\sqrt{N}} \sum_{i=1}^{I} \sum_{k=0}^{N-1} X_{i}(k) e^{\frac{j 2 \pi k n}{N}}\right] * f_{i}(n)
$$



Figure 1. F-OFDM transceiver system (transmitter-upper part, receiver-lower part)

Filtered orthogonal frequency division multiplexing for improved $5 G$ systems (Ammar A. Sahrab) 
The (1) can be seen as CP-OFDM when removing the filter $f_{i}(n)$. Hence, each individual sub-band is completely representing a separated CP-OFDM symbol. Accordingly, f-OFDM is flexible in its construction with different numerology. At the receiving end, matched filtering used to remove the effect of the $f_{i}(n)$ filter, which can be represented as,

$$
x_{r}(n)=\left\{\left[\frac{1}{\sqrt{N}} \sum_{i=1}^{I} \sum_{k=0}^{N-1} X_{i}(k) e^{\frac{j 2 \pi k n}{N}}\right] * f_{i}(n)\right\} * f_{i}^{*}(-n)
$$

That is, removing the $\mathrm{CP}$, serial to parallel achievement, time to frequency conversion using fast fourier transform (FFT), then, parallel to serial multiplexing, finally detecting the signal (de-mapping). The deployed filtering in 4G LTE signal is the sinc-function windowed by rectangular window. Hence, the sinc function is multiplied by one. Accordingly, OOB emission is recognized. On the next side, f-OFDM, in this work, made use of Hanning, Hamming, and Blackman based windowed-filters, $s_{1}(n), s_{2}(n)$, and $s_{3}(n)$, respectively [29],

$$
\begin{aligned}
& s_{1}(n)=0.5\left[1+\cos \left(\frac{2 \pi n}{p}\right)\right] \\
& s_{2}(n)=0.54+0.46 \cos \left(\frac{2 \pi n}{p}\right) \\
& s_{3}(n)=0.42-0.5 \cos \left(\frac{2 \pi n}{p}\right)+0.08 \cos \left(\frac{2 \pi n}{p}\right)
\end{aligned}
$$

where $p$ is the window size and $n=-p / 2, \ldots,-1,0,1, \ldots, p / 2$. Accordingly, windowed-sic filter can be formulated

$$
f_{i}(n)=\frac{\sin (\omega n)}{\omega n} \cdot s_{i}(n)
$$

Having described the core of the physical layer, it is worth describing the air interface structure of $5 \mathrm{G}$ systems and its difference with respect to $4 \mathrm{G}$ system. First, refer to Figure 2. There is frame structure extends to $10 \mathrm{~ms}$, each frame divided to $10 \mathrm{sub}$-frames, each is $1 \mathrm{~ms}$. Each sub-frame consists of 2-time slots, each of $0.5 \mathrm{~ms}$. there are 7 or 6 OFDM symbols in each time slot according to the CP length. This description is shown in Figure 2 as the time-axis. The vertical axis of Figure 2 represents the frequency-domain representing the OFDM subcarriers. Each 12-adjucent subcarriers constitute the resource block (RB) with duration of $0.5 m s$ (one-time slot). The resource element (RE), on the other hand, is the smallest part in the resource grid (RG) shown in Figure 2, where one RE is consists of one OFDM symbol. It can be seen that there are different dimensions in the RG; this is the main con in the $5 \mathrm{G}$ flexible structure of the RBs. That is, according to $\Delta \mathrm{f}=15 \mathrm{KHz} \times 2 \mu$, the subcarrier spacing will be $\Delta \mathrm{f}=15 \mathrm{KHz}, 30 \mathrm{KHz}, 60 \mathrm{KHz}, 120 \mathrm{KHz}$, and $240 \mathrm{KHz}$ [30]. In $4 \mathrm{G}$ systems, subcarrier spacing is fixed to $15 \mathrm{KHz}$; therefore, $4 \mathrm{G} \mathrm{RG}$ is not as flexible as $5 \mathrm{G}$ system.

The link level simulation is that the physical downlink shared channel (PDSCH) will be constructed (using properly designed toolbox in MATLAB) to determine the achieved throughput. Figure 3 depicts the link level round-trip in this work. First, binary data coded to the transport shared channel, then it will be coded to the PDSCH where the demodulation reference symbol (DM-RS) will be generated and synchronization signal (SS); primary and secondary (PSS, SSS) respectively, are also mapped in the PDSCH. Then, precoding achieved before the different numerologies employed in the construction of the CP-OFDM/f-OFDM block.

Clustered delay line (CDL) channel will be the medium to propagate the signal with additive white gaussian noise (AWGN) as shown in Figure 3. At the receiver, timing/synchronization is the first step accomplished to synchronize the transmitter with the receiver, then demodulation according to the used modulation type in the transmitter (CP-OFDM or f-OFDM). After that, channel estimation [31] is used to detect and decode the PDSCH, from which the DL-SCH transport channel can be deduced. Finally, the binary data will be outputted. However, the hybrid automatic repeat-request (HARQ) is used to find if it is required to retransmit the signal again by detecting the cyclic redundancy check code of the last transmitted signal as shown in Figure 3 [30]. In the next section, simulation results will be evaluated for a specific parameter according to the standards of the third generation partnership project (3GPP) [30]. 


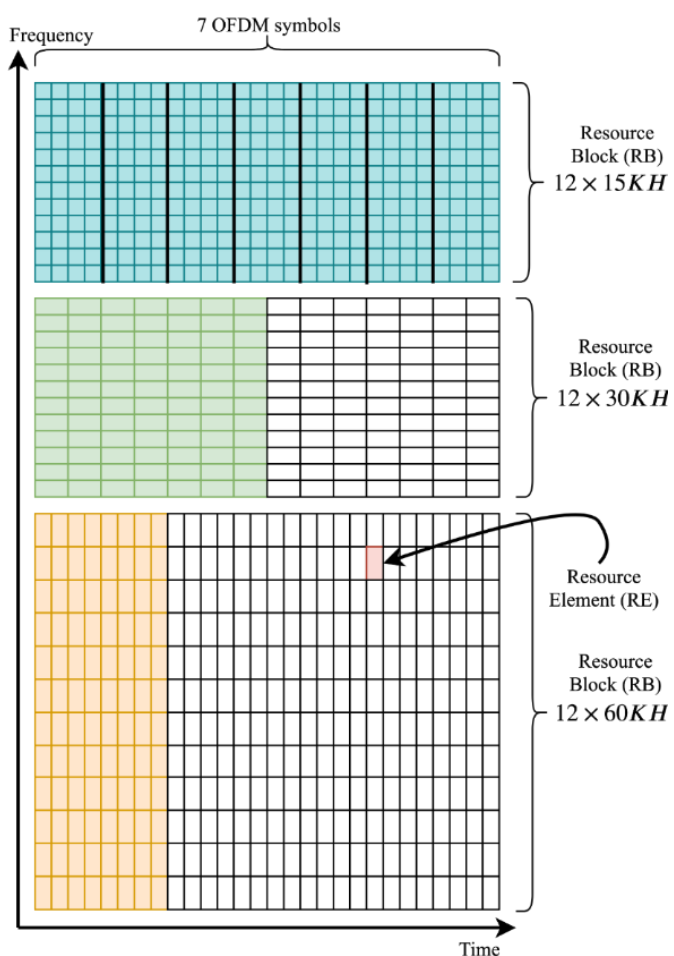

Figure 2. 5G air interface structure showing different numerologies

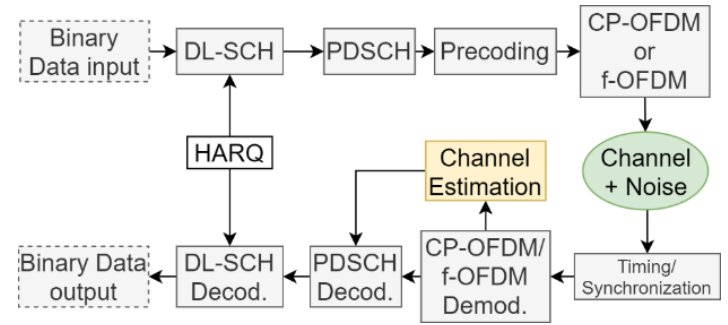

Figure 3. Physical downlink shared channel (PDSCH) link-level transceiver chain structure

\section{RESULTS AND DISCUSSION}

To be a fair comparison between CP-OFDM and f-OFDM, the subcarrier spacing is fixed to 30 $\mathrm{KHz}$, both waveforms have the same $\mathrm{CP}$ length, and number of $\mathrm{RBs}=51$, each consists of 12 subcarriers, two receive antennas and eight transmit antennas, consisting $8 \times 2$ MIMO system, without perfect channel estimation for the CDL propagation channel, as shown in Table 1.

Table 1. Setting parameters for the simulated scenario

\begin{tabular}{ll}
\hline \multicolumn{1}{c}{ Title } & \multicolumn{1}{c}{ Value settings } \\
\hline Total Number of Subcarriers $N$ & 2048 \\
Subcarrier spacing $\Delta f$ & $30 \mathrm{KHz}$ \\
Number of RB & 51 \\
Number of subcarriers per RB & 12 \\
MIMO configuration & $8 \times 2(8$-downlink $\times 2$-Uplink $)$ \\
Window/filter & CP-OFDM: rectangular window \\
& f-OFDM: Hanning, Hamming, Blackman based filtering windows \\
\hline
\end{tabular}

That is, channel estimation is accomplished using the DM-RS symbols, which are available in the PDSCH. The size of the OFDM symbol will be also fixed to both CP-OFDM and f-OFDM, N=2048 is the number of subcarriers. Number of codewords mapped in the PDSCH is one. In Figure 4 the BER 
performance comparison between CP-OFDM and f-OFDM based Hanning, Hamming, and Blackman windowed sinc filters is shown. Figure 4 is based on the 64QAM constellation mapping. While Figure 5 presents the BER performance comparison of the 256QAM for the CP-OFDM and f-OFDM waveforms.

The BER curves in Figure 4 show that Hanning based signal outperforms the other waveforms, while the other waveforms are almost identical to the performance of the CP-OFDM signal. On the other hand, the BER of the waveforms shown in Figure 5, which are based on higher order constellation mapping, are all have the same performance. This shows that at higher orders of baseband mapping, the BER did not change dramatically. To a fair comparison, it is worth to examine the EVM and MER results. Thus, the peak EVM results are $-10.2 \mathrm{~dB},-10.8 \mathrm{~dB},-12 \mathrm{~dB},-12.2 \mathrm{~dB}$ for the 64QAM CP-OFDM, Hanning, Hamming, Blackman based f-OFDM waveforms, respectively. Thus, the performance of Blackman based f-OFDM is outperforming the others with respect to CP-OFDM signal. When the constellation order became 256QAM, the EVM became $-11.9 \mathrm{~dB},-12.8 \mathrm{~dB},-13.1 \mathrm{~dB},-13.8 \mathrm{~dB}$, for the CP-OFDM, hanning, hamming, and blackman based f-OFDM waveforms, respectively. Accordingly, Blackman based waveform outperforms the others, with respect to CP-OFDM signal.

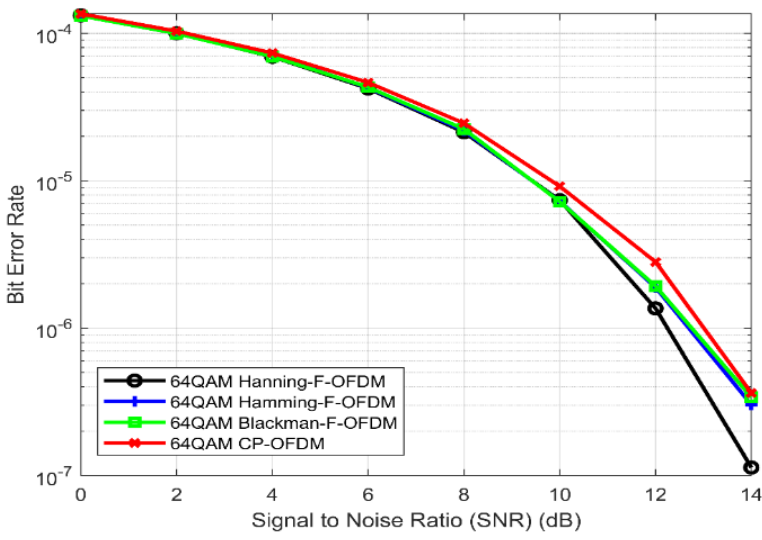

Figure 4. Comparison of 64QAM f-OFDM and CPOFDM waveforms

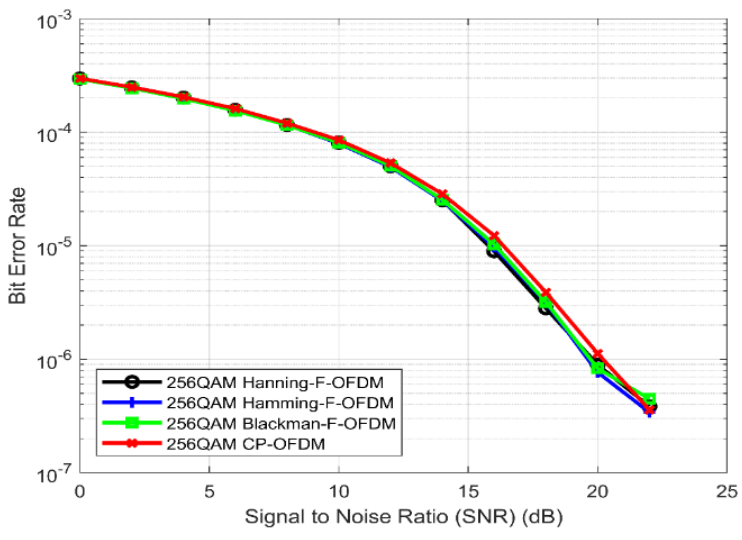

Figure 5. Comparison of 256QAM f-OFDM and CP-OFDM waveforms

The power spectral density (PSD) of the simulated signals are shown in Figure 6. It is obvious that Blackman based f-OFDM waveform has the most outperforming behavior among other f-OFDM waveforms as compared to that of CP-OFDM, where CP-OFDM has sidelobe level around $-60 \mathrm{dBW} / \mathrm{Hz}$, while Blackman based signal is very low. While Hamming based signal is about $-110 \mathrm{dBW} / \mathrm{Hz}$, which is higher than Blackman but still better than that of CP-OFDM signal.

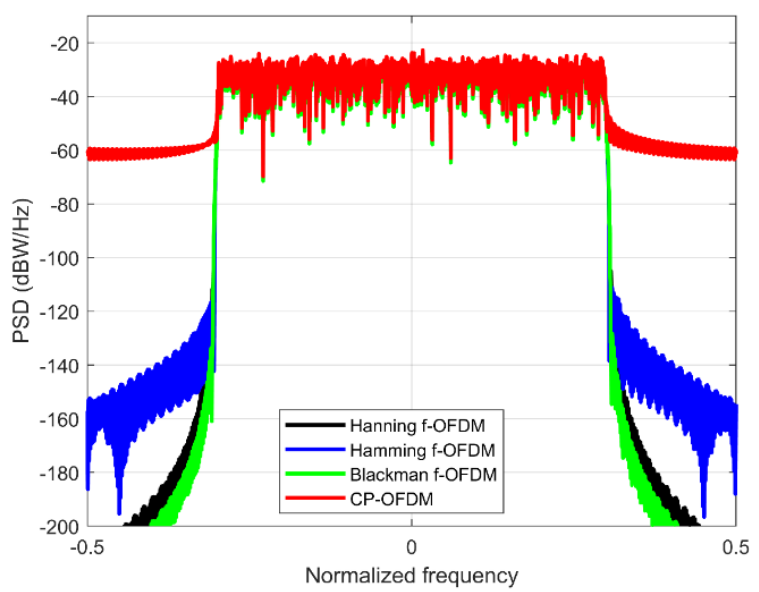

Figure 6. Comparison of the PSD of the f-OFDM and CP-OFDM waveforms 
On the other hand, the system throughput can be seen in Figure 7 where a comparison between CPOFDM, Hanning, Hamming, and Blackman based f-OFDM waveforms are drawn. It is shown that CPOFDM performance is lower than other based f-OFDM signals. The outperformed behavior was recognized for the f-OFDM waveform based Blackman windowed-sinc, where the achieved throughput was 7.25Mbps at $\mathrm{SNR}=20 \mathrm{~dB}$ based on 256QAM mapping, while the same waveform based on 64QAM mapping, the achieved throughput was 5.5Mbps. Other filtered waveforms are 5.8Mbps and 6.8Mbps for the 256QAM Hamming and Hanning based waveforms, respectively. While the same waveforms based on 64QAM, the accomplished throughputs are 4.2Mbps and 5Mbps, for the Hamming and Hanning based waveforms, respectively. These results confirm the performances shown in Figure 6, which is the PSD of these waveforms. That is, according to the PSD of CP-OFDM, the expected throughput should be the lowest among others, since the OOB emission was higher than other waveforms. While Blackman based waveform shows lowest level of OOB emission, where it was explained in the last paragraph that the PSD that Blackman based f-OFDM waveform has the most outperforming behavior among other f-OFDM waveforms.

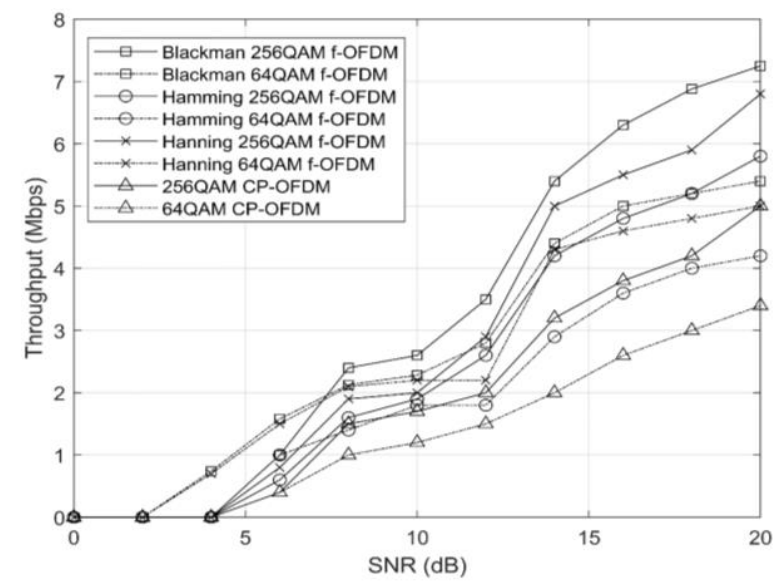

Figure 7. Throughput comparison of f-OFDM and CP-OFDM waveforms

\section{CONCLUSION}

In this paper it was shown that the throughput of the filtered based waveforms, particularly speaking, f-OFDM was improved with respect to that of CP-OFDM. However, it is concluded that the baseband constellation mapping did not put limitations on the f-OFDM based signals. Furthermore, the OOB emission was lower than the CP-OFDM, which is one of the main requirements for the $5 \mathrm{G}$ wireless systems, by which the latency has been reduced dramatically.

\section{REFERENCES}

[1] A. I. B. F. d. Almeida, L. L. Mendes, J. J. P. C. Rodrigues, and M. A. A. d. Cruz, "5G waveforms for IoT applications," IEEE Communications Surveys \& Tutorials, vol. 21, pp. 2554-2567, Apr. 2019, doi: 10.1109/COMST.2019.2910817.

[2] N. A. Mohammed, A. M. Mansoor, and R. B. Ahmad, "Mission-critical machine-type communication: An overview and perspectives towards 5G," IEEE Access, vol. 7, pp. 127198-127216, Jan. 2019, doi: 10.1109/ACCESS.2019.2894263.

[3] A. Hammoodi, L. Audah, and M. A. Taher, "Green coexistence for 5G waveform candidates: A review," IEEE Access, vol. 7, pp. 10103-10126, Jan. 2019, doi: 10.1109/ACCESS.2019.2891312.

[4] X. Zhang, L. Zhang, P. Xiao, D. Ma, J. Wei, and Y. Xin, "Mixed numerologies interference analysis and internumerology interference cancellation for windowed OFDM systems," IEEE Transactions on Vehicular Technology, vol. 67, pp. 7047-7061, Apr. 2018, doi: 10.1109/TVT.2018.2826047.

[5] ETSI.TS.138.213, "5G; NR: Physical layer procedures for control," 3GPP TS 38.213, vol. Release 15, no. version 15.6.0, 2019.

[6] J. G. Andrews, et al., "What will 5G be?," IEEE Journal on Selected Areas in Communications, vol. 32, pp. 10651082, June 2014, doi: 10.1109/JSAC.2014.2328098.

[7] P. Banelli, S. Buzzi, G. Colavolpe, A. Modenini, F. Rusek, and A. Ugolini, "Modulation formats and waveforms for 5G networks: Who will be the heir of OFDM?: An overview of alternative modulation schemes for improved spectral efficiency," IEEE Signal Processing Magazine, vol. 31, no. 6, pp. 80-93, Oct. 2014, doi: 10.1109/MSP.2014.2337391. 
[8] I. Parvez, A. Rahmati, I. Guvenc, A. I. Sarwat, and H. Dai, "A survey on low latency towards 5G: RAN, core network and caching solutions," IEEE Communications Surveys \& Tutorials, vol. 20, no. 4, pp. 3098-3130, May 2018, doi: 10.1109/COMST.2018.2841349.

[9] Y. Liu, et al., "Waveform design for 5G networks: Analysis and comparison," IEEE Access, vol. 5, pp. 1928219292, Feb. 2017, doi: 10.1109/ACCESS.2017.2664980.

[10] T. Wild, F. Schaich, and Y. Chen, "5G air interface design based on universal filtered (UF-)OFDM," In 2014 19th International Conference on Digital Signal Processing, Hong Kong, China, Aug. 2014, pp. 699-704, doi: 10.1109/ICDSP.2014.6900754.

[11] Al-Naffouri, T., Y., Islam, K., M., Z., Al-Dhahir, N., and Lu, S., "A model reduction approach for OFDM channel estimation under high mobility conditions," IEEE Transactions on Signal Processing, vol. 58, vol. 4, pp. 21812193, 2010, doi: 10.1109/TSP.2009.2039732.

[12] P. Varzakas, "Optimization of an OFDM Rayleigh fading system," International Journal of Communication Systems, vol. 20, pp. 1-7, 2007, doi: 10.1002/dac.807.

[13] R. Nissel, M. Rupp, and R. Marsalek, "FBMC-OQAM in doubly-selective channels: A new perspective on MMSE equalization," In 2017 IEEE 18th International Workshop on Signal Processing Advances in Wireless Communications, Sapporo, Japan, Dec. 2017, pp. 1-5, doi: 10.1109/SPAWC.2017.8227806.

[14] A. Farhang, N. Marchetti, and L. E. Doyle, "Low-complexity modem design for GFDM," IEEE Transactions on Signal Processing, vol. 64, no. 6, pp. 1507-1518, Nov. 2016, doi: 10.1109/TSP.2015.2502546.

[15] X. Zhang, M. Jia, L. Chen, J. Ma, and J. Qiu, "Filtered-OFDM - enabler for flexible waveform in the 5th generation cellular networks," In 2015 IEEE Global Communications Conference (GLOBECOM), San Diego, CA, USA, Dec. 2015, pp. 1-6, doi: 10.1109/GLOCOM.2015.7417854

[16] A. Sahin, I. Guvenc, and H. Arslan, "A survey on multicarrier communications: Prototype filters, lattice structures, and implementation aspects," IEEE Communications Surveys \& Tutorials, vol. 16, no. 3, pp. 1312-1338, Dec. 2014, doi: 10.1109/SURV.2013.121213.00263.

[17] M. Bellanger, et al., "FBMC physical layer : A primer," PHYDYAS, pp. 1-31, 2010.

[18] A. Bedoui, and M. Et-tolba, "A comparative analysis of filter bank multicarrier (FBMC) as 5G multiplexing technique," In 2017 International Conference on Wireless Networks and Mobile Communications (WINCOM), Rabat, Morocco, Nov. 2017, pp. 1-7, doi: 10.1109/WINCOM.2017.8238200.

[19] J. Abdoli, M. Jia, and J. Ma, "Filtered OFDM: A new waveform for future wireless systems," In 2015 IEEE 16th International Workshop on Signal Processing Advances in Wireless Communications (SPAWC), Stockholm, Sweden, June 2015, pp. 66-70, doi: 10.1109/SPAWC.2015.7227001.

[20] J. Li, K. Kearney, E. Bala, and R. Yang, "A resource block based filtered OFDM scheme and performance comparison," In International Conference on Telecommunications (ICT 2013), Casablanca, Morocco, Oct. 2013, pp. 1-5, doi: 10.1109/ICTEL.2013.6632084.

[21] A. Idris, N. A. M. Deros, I. Taib, M. Kassim, M. D. Rozaini, and D. M. Ali, "PAPR reduction using huffman and arithmetic coding techniques in F-OFDM system," Bulletin of Electrical Engineering and Informatics, vol. 7, pp. 257-263, June 2018, doi: 10.11591/eei.v7i2.1169.

[22] M. A. Taher, H. S. Radhi, and A. K. Jameil, "Enhanced F-OFDM candidate for 5G applications," Journal of Ambient Intelligence and Humanized Computing, vol. 12, pp. 635-652, 2021, doi: 10.1007/s12652-020-02046-3.

[23] J. Bazzi, P. Weitkemper, K. Kusume, A. Benjebbour, and Y. Kishiyama, "Design and performance tradeoffs of alternative multi-carrier waveforms for 5G," In 2015 IEEE Globecom Workshops (GC Wkshps), San Diego, CA, USA, Dec. 2015, pp. 1-6, doi: 10.1109/GLOCOMW.2015.7414010.

[24] C. An, B. Kim, and H. Ryu, "WF-OFDM (windowing and filtering OFDM) system for the 5G new radio waveform," In 2017 IEEE XXIV International Conference on Electronics, Electrical Engineering and Computing (INTERCON), Cusco, Peru, Aug. 2017, pp. 1-4, doi: 10.1109/INTERCON.2017.8079635.

[25] S. Gokceli, E. Basar, and G. K. Kurt, "Universal filtered OFDM with filter shift keying - invited paper," In 2018 IEEE 87th Vehicular Technology Conference (VTC Spring), Porto, Portugal, June 2018, pp. 1-5, doi: 10.1109/VTCSpring.2018.8417532.

[26] F. A. P. d. Figueiredo, N. F. T. Aniceto, J. Seki, I. Moerman, and G. Fraidenraich, "Comparing f-OFDM and OFDM performance for MIMO systems considering a 5G scenario," In 2019 IEEE 2nd 5G World Forum (5GWF), Dresden, Germany, Germany, Sep. 2019, pp. 532-535, doi: 10.1109/5GWF.2019.8911702.

[27] H. Chen, J. Hua, F. Li, F. Chen, and D. Wang, "Interference analysis in the asynchronous f-OFDM systems," IEEE Transactions on Communications, vol. 67, no. 5, pp. 3580-3596, May 2019, doi: 10.1109/TCOMM.2019.2898867.

[28] H. Kim, Y. Park, J. Kim, and D. Hong, "A low-complex SVD-based F-OFDM," IEEE Transactions on Wireless Communications, vol. 19, no. 2, pp. 1373-1385, Feb. 2020, doi: 10.1109/TWC.2019.2953540.

[29] F. J. Harris, "On the use of windows for harmonic analysis with the discrete Fourier transform," in Proceedings of the IEEE, vol. 66, pp. 51-83, Jan. 1978, doi: 10.1109/PROC.1978.10837.

[30] ETSI.TS.138.211, "5G; NR; Physical channels and modulation," in 3rd Generation Partnership Project; Technical Specification Group Radio Access Network, vol. (3GPP TS 38.211 version 15.2.0 Release 15, 2018.

[31] P. Manhas, and M. K. Soni, "Performance of OFDM system under different fading channels and channel coding," Bulletin of Electrical Engineering and Informatics, vol. 6, no. 1, pp. 54-61, Mar. 2017, doi: 10.11591/eei.v6i1.591. 


\section{BIOGRAPHIES OF AUTHORS}
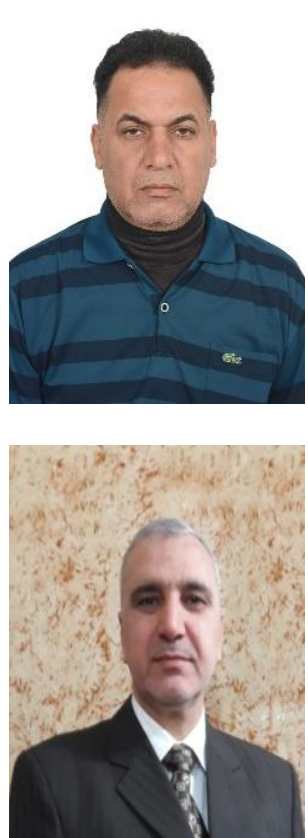

Alaa Doohee Yaseen recived his B.Sc. degree in Electrical Engineering from AlMustansiriyah University, Faculty of Engineering, Baghdad, Iraq, in 2007. He obtained his M.Sc. degree in Electronics \& Communications Engineering from Al-Mustansiriyah University, College of Engineering, Baghdad, Iraq, in 2020. He worked as a medical instrumentation engineer with the ministry of helth-Iraq, then with Huawei-Iraq as a communication field engineer. Currently he is with Erricson as a communication field engineer. His field of interest is Mobile communications engineering, digital communication engineering, $4 \mathrm{G}$ and $5 \mathrm{G}$.

Ammar Ali Sahrab received his B.Sc. degree in Electrical Engineering (Honours) from AlMustansiriyah University, Faculty of Engineering, Baghdad, Iraq, in 1997. He obtained his M.Sc. degree in Electronics \& Communications Engineering from Al-Mustansiriyah University, College of Engineering, Baghdad, Iraq, in 2000. After that, he obtained Ph.D. degree in Electronics, Telecommunications and Information Theory from University "Politehnica" of Bucharest, The Faculty of Electronics, Telecommunications and Information Technology, Bucharest, Romania, in 2016. He currently holds the position of Associate Professor in the department of Electrical Engineering at AlMustansiriyah University. His research interests include: Mobile Communications, IoT based on microcontroller, Antennas Design and Implementation 